\title{
Rattraper le nouvel Occident : « L'initiative d'excellence » allemande, les études régionales et la re-production des inégalités
}

Alemania : Excelencia alemana y Areas de Estudios o como traer al eurocentrismo por la puerta de atrás.

Catching Up with the (New) West: German "Excellence Initiative," Area Studies and the Re-Production of Inequality

\section{Manuela Boatcă}

Traducteur : Laura Pertuy

\section{(2) OpenEdition} Journals

Édition électronique

URL : https://journals.openedition.org/ideas/243

DOI : 10.4000/ideas.243

ISSN : 1950-5701

Éditeur

Institut des Amériques

Référence électronique

Manuela Boatcă, « Rattraper le nouvel Occident : «L'initiative d'excellence » allemande, les études régionales et la re-production des inégalités », IdeAs [En ligne], 2 | 2012, mis en ligne le 16 juillet 2012, consulté le 19 octobre 2022. URL : http://journals.openedition.org/ideas/243 ; DOI : https://doi.org/ $10.4000 /$ ideas. 243

Ce document a été généré automatiquement le 19 octobre 2022

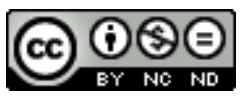

Creative Commons - Attribution - Pas d'Utilisation Commerciale - Pas de Modification 4.0 International - CC BY-NC-ND 4.0

https://creativecommons.org/licenses/by-nc-nd/4.0/ 


\title{
Rattraper le nouvel Occident : « L'initiative d'excellence » allemande, les études régionales et la re-production des inégalités
}

\author{
Alemania: Excelencia alemana y Areas de Estudios o como traer al \\ eurocentrismo por la puerta de atrás. \\ Catching Up with the (New) West: German "Excellence Initiative," Area Studies \\ and the Re-Production of Inequality
}

Manuela Boatcă

Traduction : Laura Pertuy

Je remercie Alanna Lockward et les trois relecteurs anonymes pour leurs suggestions et commentaires précieux sur cet article.

L'étiquette "d'occidentalisation", que sa connotation soit positive ou négative, a été grandement utilisée pour décrire, ou déplorer, la convergence de modèles sociaux, économiques, politiques ou culturels au sein d'un modèle occidental soi-disant homogène. Cependant, ce qui valait auparavant comme "occidental" a considérablement changé au fil du temps et de l'espace mais aussi en fonction des changements dans les relations de pouvoir entre les parties du monde, lesquelles ont modelé la notion "d'occident" et en ont fait une référence normative et un modèle à suivre $^{1}$. En ce qui concerne les systèmes d'éducation supérieure, la mention "d'université occidentale", étiquette utilisée par définition dans des contextes nonoccidentaux à qui elle servait de modèle, s'est successivement référée à l'Université de la Renaissance en Italie, l'Espagne et le Portugal au XVII siècle, à l'Université des Lumières en Angleterre, en Allemagne et en France à partir de la fin du XvIII ${ }^{e}$ siècle, tout comme à l'Université de la guerre froide aux États-Unis, laquelle, sponsorisée par les militaires, s'orientait grandement vers la politique étrangère (Mignolo 2003; Wallerstein 1997). 
2 Le système d'enseignement supérieur allemand a particulièrement modelé la définition moderne de l'université occidentale pendant une période décisive de son histoire. Les réformes du début du XIX ${ }^{\mathrm{e}}$ siècle initiées par Wilhelm Von Humboldt et concentrées sur l'unification de la recherche et de l'enseignement dans des institutions sponsorisées par l'état, encouragèrent la restructuration de l'éducation supérieure à travers l'Europe en suivant l'idée d'un soutien de l'état à la fois pour l'éducation et pour la science dans les structures universitaires, plutôt qu'au sein d'institutions spécifiques dépendant d'un mécénat privé, ce qui était le cas jusqu'au XviII siècle. La connaissance que les institutions d'éducation supérieure ont d'elles-mêmes et leur relation aux états-nations émergents en Europe occidentale, les principaux parti-pris méthodologiques tout comme la démarcation concordante des disciplines académiques aussi bien en Grande Bretagne qu'en France, autres acteurs majeurs de l'établissement de l'Université au $\mathrm{XIX}^{\mathrm{e}}$ siècle, prirent forme sous l'influence ou en confrontation directe avec l'idée d'Humboldt d'un appareil étatique éducationnel (Kulturstaatskonzept), de sciences de l'État (Staatswissenschaften), d'historicisme allemand, et de Methodenstreit entre les approches idiographiques et nomothétiques de la production de savoir scientifique (DiMeglio 2004; Mielants 2004). Avec l'ascension des États-Unis vers un statut hégémonique au sein du système-monde après la Seconde Guerre mondiale, le modèle de l'université Humboldtienne, qui avait prévalu tout au long du xix siècle et dans une majeure partie $\mathrm{du} \mathrm{xx}^{\mathrm{e}}$ siècle, perdit progressivement du terrain pour adopter le modèle américain, qui devint ainsi l'épitomé de l'université occidentale à l'aube du xxI siècle.

3 La perte historique du pouvoir d'établir des standards académiques revient de façon récurrente dans l'université comme dans la législation allemande ; la compétition avec le système américain en est un rappel constant. Que la réputation des universités américaines d'aujourd'hui corresponde à celle des universités allemandes du XIX siècle et du début du $\mathrm{xx}^{\mathrm{e}}$ siècle est tour à tour dénoncé, déploré, ou utilisé comme preuve de la mauvaise mise en application de la dernière réforme de l'éducation supérieure par l'état allemand ; mais dans l'état, elle est incontestée (Roche 2010 ; Darnstädt 2010). On peut dire de même de la fuite des cerveaux qui a placé l'Allemagne en tête de liste parmi les pays dont les étudiants effectuent leur doctorat aux États-Unis et dans les états membres de l'Union Européenne. Cela a entrainé des contre-mesures spécifiques et des homecoming programs (programmes de récupération de ces mêmes doctorants) en vigueur depuis 2006 et mis en place par l'association des universitaires allemands (Jung 2010). En résulte, comme dans le cas d'autres systèmes universitaires européens à la recherche de leur splendeur universitaire passée, une occidentalisation renouvelée, cette fois conçue sur le modèle américain.

4 Cet essai développe la thèse selon laquelle la politique d'éducation supérieure actuellement menée par l'Allemagne représente en fait un processus de rattrapage institutionnel qui, sans le vouloir, reproduit les principales erreurs des politiques de modernisation visant l'occidentalisation au milieu du $\mathrm{xx}^{\mathrm{e}}$ siècle, tout autant que leurs principales conséquences engendrées par la reproduction de structures d'inégalité déjà existantes. En même temps, étant donné qu'il s'agit ici d'une compétition interne entre les Etats-Unis et l'Europe occidentale plus généralement, et les Etats-Unis et l'Allemagne plus particulièrement, la norme académique en cours de négociation demeure ancrée dans la logique capitaliste qui caractérise l'université occidentale depuis le début de la période moderne, et qui, de plus, la renforce. En se focalisant sur le regain d'occidentalisation du système universitaire allemand, cet essai se divise en 
deux étapes : dans un premier temps, il examine comment le nouveau discours d'élite de l'éducation supérieure allemande, encouragé par des mesures telles que la récente “initiative d'excellence", produit de nouvelles structures d'exclusion. Ensuite, l'essai se penche sur le renouveau des études régionales comme l'une des principales cibles de l'initiative d'excellence et du financement de l'université. Cela a pour but de montrer comment la politique, derrière le récent soutien financier de l'état à l'étude des régions non occidentales, appuie les principales hypothèses de la théorie de la modernisation et reproduit les asymétries de la production de la connaissance caractéristique des approches euro-centrées et état-centrées des problèmes en question. L'essai s'achève sur une comparaison entre les processus d'institutionnalisation d'opinions subalternes entre les États-Unis et l'Allemagne.

\section{Le conte de deux occidents : les États-Unis contre le système universitaire allemand}

5 Si les universitaires et législateurs allemands, tout comme leurs homonymes à travers l'Europe occidentale, déplorent aujourd'hui l'américanisation de leur système universitaire, un processus similaire voire inverse de dés-allemanisation de l'éducation supérieure (DiMeglio 2004) prit place aux États-Unis au début du Xx siècle.

6 Aux prises avec un rapide développement capitaliste depuis la guerre de Sécession, mais aussi avec des structures étatiques faibles et non armées pour gérer la "question sociale" émergente, les États-Unis, contrairement aux états européens occidentaux avant eux, furent témoins d'une forte croissance des universités privées plus que des universités publiques, les premières étant financées grâce à la fortune de magnas ${ }^{2} \mathrm{de}$ l'industrie. Comme l'éducation supérieure était proche de l'entreprise, ses promoteurs, dont beaucoup étaient des scientifiques sociaux formés principalement dans l'Allemagne du xix ${ }^{e}$ siècle, abandonnèrent peu à peu la tradition de pensée distinctement allemande qui se concentrait sur l'historicité, le holisme et la synthèse disciplinaire en faveur du scientisme, du positivisme et de l'empirisme, laquelle était dictée par la montée des sciences naturelles dans le contexte du capitalisme industriel (Manicas 1991; DiMeglio 2004). L'université inspirée du modèle allemand, c'est-à-dire organisée autour de chaires individuelles, fut vite remplacée par une structure composée de départements aux multiples professeurs, mieux adaptée au grand nombre d'étudiants aux États-Unis et plus propice à la création de réseaux scientifiques dont la recherche attira d'importants fonds financiers venant des entreprises (Stremlin 2004). L'émergence du modèle de l'université américaine et son internationalisation après la Première Guerre mondiale se bâtit ainsi sur une dés-allemanisation des structures universitaires en général et des sciences sociales américaines en particulier. Grâce à l'afflux de scientifiques européens occidentaux vers les États-Unis pendant la Seconde Guerre mondiale et notamment la montée drastique des fonds financiers gouvernementaux dédiés à la recherche à des fins militaires pendant la guerre froide, les universités de recherche américaines s'affirmèrent comme les leaders de la culture scientifique mondiale.

7 À son tour, l'ensemble du système éducatif allemand souffrit d'une série de coups durs (générés, en grande partie, par lui-même). Le modèle même du soutien de l'état à l'éducation et à la science, lequel avait entraîné le premier exemple d'école obligatoire au début du XVIII ${ }^{\mathrm{e}}$ siècle en Prusse, mena à une structure hiérarchique en trois tranches 
dans l'Allemagne de la fin du XIXe siècle. Alors que des fonctionnaires étaient recrutés dans la tranche supérieure et recevaient ainsi une éducation générale (ce qui ensuite leur assurait un accès à l'éducation supérieure), la formation professionnelle devint un domaine éducatif à part, plus rattaché aux classes inférieures (Gottschall/Hagemann 2002). Dès les années 60 , la haute sélectivité du système éducatif allemand, son inhabilité à exploiter le potentiel d'apprentissage de la strate sociale inférieure et son apport financier globalement maigre lui valurent l'étiquette de "catastrophe éducative allemande" (Picht 1964) et une réputation peu favorable parmi les nations industrialisées. L'expansion qui s'ensuivit dans l'éducation secondaire comme dans l'éducation supérieure réussit à égaliser certaines de ses inégalités horizontales, notamment eu égard au sexe, mais pas les inégalités verticales telles que l'origine sociale, c'est-à-dire la classe ${ }^{3}$. Bien que la restriction de l'éducation supérieure à la formation des élites nationales, vraie pour la plupart des pays européens occidentaux, a mené à des stratégies nationales distinctes de différenciation parmi les types d'éducation supérieure (Paradeise et al. : 2001) Les résultats du système allemand, favorisant les inégalités, se sont montrés particulièrement persistants : les résultats lamentables de l'évaluation PISA 2002, plaçait les jeunes allemands de 15 ans (testés sur leur compréhension écrite, les mathématiques et les sciences naturelles) bien endessous de la moyenne de l'OCDE à tous points de vue. L'évaluation révélait également des difficultés structurelles empêchant l'avancée éducative de la classe inférieure et des enfants immigrés, ce qui a montré que les accusations des années 60 étaient encore valables dans les années 2000. Avec le nombre grandissant des abandons à l'université et l'âge comparativement élevé des étudiants en deuxième cycle universitaire ${ }^{4}$, de tels résultats rendirent les qualificatifs dont s'enorgueillissait l'Allemagne, parmi lesquelles "la terre des poètes et des penseurs" et "la nation de l'éducation" (Bildungsnation), de plus en plus incorrectes et le "retard mental" de son système scolaire un sujet de crainte grandissant (Smolka 2002; Gottschall/Hagemann 2002).

8 La réforme de l'université de Bologne, destinée à créer une "zone européenne d'éducation supérieure» de comparabilité mais aussi de compétitivité accrues au niveau international par la réduction de la durée des études universitaires, la diminution du taux d'abandon des études, et l'amélioration de l'accès à l'emploi pour les diplômés, paraissait être le meilleur remède pour certaines des maladies allemandes chroniques. Cependant, la voie rapide de trois ans jusqu'à la licence devenait prédominante sur le transfert de savoir et le rendement annuel d'étudiants prêts pour l'emploi décidait de plus en plus du sort des universités sur le marché de l'éducation supérieure. Fusèrent alors contre les législateurs allemands, les accusations d'avoir remplacé la république de la connaissance par «l'université entrepreneurial» (Helbrecht 2007), et d'avoir ainsi préféré «Siemens à Humboldt » (Darnstädt 2010), furent dirigées contre les législateurs allemands ${ }^{5}$.

9 L'idée d'associer investissements et rendements dans l'éducation et la recherche pour fournir une éducation en accord avec les besoins du système économique, est apparue pour la première fois dans les années 80 (Goedegebuure et al. 1993). Des recherches récentes ont également montré que l'approche entrepreneuriale de la gouvernance de l'université s'est progressivement installée en Europe occidentale sur les deux dernières décennies, et plus rapidement depuis les années 90 (Paradeise et al. 2009: 225). Ce qui suit explore en détail les configurations spécifiques auxquelles cette mouvance a donné naissance dans le contexte de l'initiative d'excellence allemande. 


\section{L'initiative d'excellence : un succès ?}

10 de l'université de Bologne engageait aussi une différenciation croissante entre les universités qui pouvaient répondre aux besoins du nouvel appareil entrepreneurial et celles qui ne le pouvaient pas. En fonction de la réputation, de la taille de l'université ou de l'UFR, des fonds de l'état disponibles, ou d'une situation géographique attrayante, la capacité à mettre en place des programmes de licence (mais non de master ou de doctorat) impliquait la plupart du temps un revirement vers le statut de "simple " université d'enseignement. Bien que souvent saluée pour avoir mis fin à la «fiction de l'égalité » qui gouvernait l'éducation supérieure en Allemagne, la politique qui visait à extraire l'enseignement des institutions de recherche, modelées sur l'exemple américain dans l'espoir de reproduire le succès des universités de la Ivy League, ne produisit pas les mêmes résultats. En établissant, d'un côté, de prétendues « universités d'élite » qui bénéficient de considérables fonds de l'état pour «l'excellence de leur recherche ", et en confinant de l'autre côté l'écrasante majorité d'étudiants dans des « universités de masse » (déjà) bondées où la charge d'enseignement n'alloue presque aucun temps libre pour la recherche, de telles mesures politiques dessinent une forte ligne imaginaire entre les universités qui, ayant été structurées selon l'idéal d'Humboldt "d'unité de recherche et d'enseignement», ne tombent souvent pas strictement dans l'une ou l'autre de ces catégories (Kreckel 2008 :6).

11 La prétendue "initiative d'excellence", processus d'évaluation lancé pour la première fois entre 2006 et 2007, au cours de laquelle neuf universités, l'université libre de Berlin, l'université de Munich et l'université de Heidelberg, entre autres, furent désignées comme des " universités d'élite », représente jusqu'ici l'exemple le plus clair et le plus institutionnalisé d'une politique d'état destinée à limiter la recherche à des institutions spécialisées. Les critères de sélection, tels que les indices de publications et l'importance des allocations de bourse sur la base de la performance antérieure tout comme du potentiel innovateur, a été dénoncé comme vague et favorisant les universités déjà éminentes et bien dotées financièrement (Hombostel 2008). Des avancées plus anciennes dans cette direction, comme la création des instituts MaxPlanck et Leibniz mais aussi de la société Fraunhofer, espaces uniquement dédiés à la recherche dans les années 80 et 90, avaient seulement généré un plus large choix d'options d'emplois pour les chercheurs que ce que le système universitaire de l'état permettait alors. En contraste, la nouvelle division académique du travail initiée avec la réforme de Bologne et emmenée un pas plus loin par «l'initiative d'excellence » a servilement suivi le modèle américain en créant une rupture entre la recherche et l'enseignement, et ce au sein de la structure universitaire elle-même.

12 Avec le but explicite de "promouvoir des projets de recherche et des institutions remarquables » tout comme de "rendre la science et la recherche allemandes plus visibles au sein de la communauté scientifique ", l'initiative d'excellence, dirigée par le fonds allemand pour la recherche (DFG) avec le concours des gouvernements fédéraux et étatiques, consiste en trois lignes de financement distinctes: les formations de deuxième cycle, vouées à promouvoir de jeunes scientifiques et chercheurs; les clusters d'excellence, dédiés à approfondir la recherche de pointe en faisant marcher de concert universités, institutions non-universitaires, commerce et industrie sur des thèmes 
particulièrement prometteurs; et les stratégies institutionnelles, conçues comme un moyen de proposer une recherche de haut niveau a l'échelle universitaire. Au terme des deux premiers tours d'évaluation en octobre 2006 et octobre 2007, 85 institutions furent sélectionnées en accord avec ces directives. Parmi elles, 39 formations de deuxième cycle, 37 clusters d'excellence, et 9 «universités d'élite», autrement dit celles avec les meilleures stratégies institutionnelles (voir schéma 1). Toutes ces institutions reçoivent des fonds de 1,9 milliard d'euros étalés sur une période de cinq ans. Le deuxième tour des sélections, actuellement en cours, allouera 2,7 milliards d'euros en plus pour la période allant de 2012 à 2017. Cependant, la majeure partie de cette allocation (jusqu'à 14 millions d'euros par institution et par année) est destinée aux universités d'élite, dont les stratégies institutionnelles de développement futur promettent un renversement, de la « structure universitaire classique » à la «structure de gestion moderne " (Université libre de Berlin), établissant ainsi des "structures de gestion exécutive innovantes au niveau du conseil de l'université et du corps professoral» (RWTH Aachen). Ces stratégies envisagent même «l'université entrepreunariale" tout court (TU Munich) (DFG 2008). Selon les universitaires allemands critiques, ce changement discursif de l'euphémisme académique traditionnellement caractéristique des universités d'Europe occidentale vers une langue élitiste (désignée en termes de marketing et de publicité) est symptomatique d'une commercialisation indirecte des universités européennes (toujours financées et contrôlées par l'état, dans la majeure partie des cas), contrairement à la mercantislisation directe du travail universitaire aux États-Unis dans le contexte du financement massif des universités privées par l'entreprise (Kreckel 2006). Selon les promoteurs de l'initiative d'excellence, cela dit, le bilan de telles politiques de rattrapage est exceptionnellement positif, comme le note le site internet du fonds allemand pour la recherche :

13 Le portail vidéo incarne une success story qui a permis d'accomplir beaucoup de choses en très peu de temps, et qui poursuivra sur cette lancée dans les années à venir. Quand la science et la politique allemandes se sont unies en 2005 pour lancer l'initiative d'excellence, elles se sont données un but formidable à atteindre. Leur objectif était d'organiser une compétition pour renforcer de façon solide la recherche dans les universités allemandes et d'augmenter la visibilité de la science et de la recherche allemandes vis-à-vis de nos concurrents internationaux. Il s'agissait en effet d'objectifs ambitieux, précisément puisqu'ils engageaient de quitter la conception longtemps chérie et irrémédiablement mauvaise selon laquelle toutes les universités sont égales et devraient ainsi être traitées également. A la place, l'initiative d'excellence a poursuivi un chemin d'inégalités et un financement des élites. (DFG 2010, je souligne). 


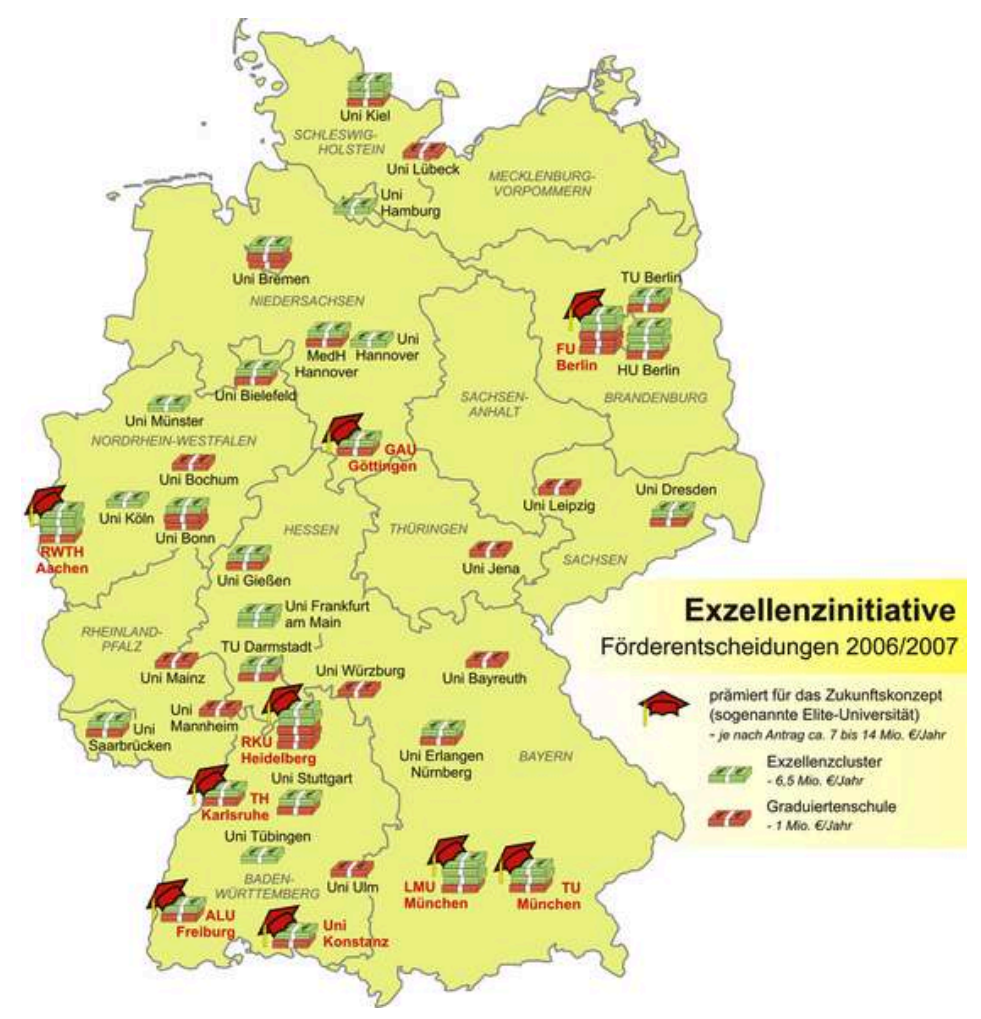

Source : DFG 2010

Ainsi, le nouveau discours élitiste de l'éducation supérieure allemande n'encourage pas seulement l'émergence de nouvelles structures d'exclusion intra-pays, mais se base aussi sur un aveu explicite de ce qui est vu comme le chemin vers le succès dans un monde à tendance mondialisante : le fait de se remettre dans la course. Tout comme une politique de modernisation institutionnelle suivant un modèle de séquences par étapes, cela laisse la possibilité de décider des conséquences sociales d'inégalités grandissantes pour une "embellie future" : l'excellence académique allemande. En fait, le discours porte les caractéristiques claires d'une modernisation au-delà de l'effort académique en tant que tel, car il essaye de traduire ses prétendus succès en politique groupée de transformation sociale. Le président du DFG, Matthias Kleiner, ingénieur de formation, l'exprime ainsi :

"[...] L'initiative d'excellence n'est pas seulement en train de changer la science et le système de recherche allemands, elle tire tout le pays vers l'avant. Elle crée des milliers d'emplois de grande qualité, elle promeut nos spécialistes, nos experts et nos dirigeants de demain. Elle contribue également à l'innovation dans les affaires et dans l'industrie. Enfin, elle montre comment la science soutient la société." (DFG $2008: 11)$

15 Comme dans la pensée classique modernisante, la politique éducative et l'importante politique de transformation sociale qu'elle prétend engendrer, reposent sur l'idéalisation d'un modèle préétabli à suivre : les standards de la Ivy League en termes d'éducation, et les États-Unis dans leur ensemble en termes de développement sociétal. Mais elles reposent également sur une certaine indifférence vis-à-vis de l'émergence et du succès de ces derniers points, comme les critiques de l'initiative d'excellence l'ont souligné de manière convaincante :

Son idéal et son point de référence se basent sur ce que le public allemand pense connaitre sur les universités américaines et seulement sur ce qu'il veut savoir à son 
sujet. C'est là le regard rayonnant que ce public adresse aux États-Unis, qui non seulement fait briller la réalité mais qui est aussi peu disposé à reconnaître sa spécificité au sein du monde occidental [...] La notion dominante souligne que la mondialisation mène à une société mondiale qui parle anglais et pense américain. Et que sa force moteur est une compétition qui ne doit pas être entravée et dont les résultats doivent être mesurables quantitativement sur le court terme. C'est la société mondialisée à laquelle l'Allemagne doit être formée. (Meyer 2010 : 568, ma traduction)

On ne fait que très peu de cas du constat selon lequel les budgets des meilleures universités américaines surpassent de beaucoup même ceux des universités allemandes d'élite les mieux dotées, dont les fonds sont attribués sur des périodes de cinq ans. Tout comme le fait que les frais universitaires annuels des universités américaines de la Ivy League qui sont plus élevés que le salaire annuel moyen aux Etats-Unis ${ }^{6}$, ne soient même pas comparables aux 500 euros par semestre de frais universitaires récemment introduits en Allemagne. Ce changement a d'ailleurs rencontré de vastes protestations étudiantes, étant donné la longue tradition allemande d'éducation "gratuite" (c'est-àdire financée par l'état) à tous les niveaux. L'introduction de frais universitaires (bien que peu élevés) pave le chemin pour des inégalités d'opportunité contre lesquelles les étudiants allemands ont protesté. Ces inégalités influencent le système d'éducation supérieure américain depuis la fin des années 70 : elles ont conduit à l'émergence d'une industrie entière de prêts universitaires, ont encouragé la croissance exponentielle de la dette étudiante, et, paradoxalement, des taux plus élevés d'abandon universitaire. De plus, ces mêmes inégalités, en faisant du travail à plein temps un pré-requis obligatoire pour pouvoir s'offrir des études universitaires, ont augmenté l'âge des étudiants américains, dont la moyenne est maintenant de 26 ans, rattrapant ainsi l'Allemagne (Donoghue 2010). Alors que les universités d'élite dont sortent d'excellents professionnels deviennent de plus en plus hors de prix (à l'exception de quelques unes) et que les universités publiques anciennement prestigieuses se replient sous des subventions de l'état en baisse, on assiste à une prolifération d'universités techniques et de community colleges (établissement de premier cycle universitaire) qui fournissent des enseignements pratiques et professionnels. N'en sortent pas des chercheurs de haute volée mais des employés du secteur tertiaire, titulaires de deux ans d'éducation supérieure (Donoghue 2010 : 635). Ainsi, pris dans son intégralité et bien longtemps après son apogée, le système d'éducation supérieure que l'Allemagne se bat pour mettre en place produit, depuis des décennies, les résultats mêmes que le système universitaire allemand a identifié comme ses points les plus faibles.

\section{Renouveau des études régionales}

Cependant, c'est "l'âge d'or" du système universitaire américain qui entraina la résurgence des études régionales, l'une des cibles les plus éminentes de l'initiative d'excellence et du financement universitaire en Allemagne. La conscience du besoin de promouvoir la recherche sur les régions du monde fut encouragée par des considérations stratégiques similaires à celles des États-Unis dans les années 40 et 50, où l'institutionnalisation des études régionales fut mise au premier plan d'un point de vue géopolitique et servit directement à la politique étrangère des États-Unis, alors en pleine guerre froide. Dans ce contexte politique, d'importantes sommes de fonds fédéraux tout comme des bourses de recherche issues de fondations majeures, furent 
attribuées pour l'étude de zones non-occidentales, particulièrement les zones communistes, et pour la protection des nouveaux états décolonisés d'Asie et d'Afrique menacés de tomber sous influence soviétique (Wallerstein 1997). Nourries par la théorie de la modernisation, les études régionales du milieu $\mathrm{du} \mathrm{xx}^{\mathrm{e}}$ siècle étaient dirigées, dans un premier temps, vers la mise en place de politiques de développement sur le modèle des États-Unis.

Leur contrepartie allemande était les "sciences régionales" (Regionalwissenschaften) de l'ancienne RDA, elles-mêmes sous l'égide idéologique du régime socialiste, mais en tant que telles (paradoxalement) moins caractérisées par un programme politique commun avec un grand penchant développementaliste que par une orientation scientifique sociale interdisciplinaire. En effet, parmi les nombreuses disciplines régionales enseignées en Allemagne de l'est, seules celles concentrées sur la Chine (Chinawissenschaften) peuvent rétrospectivement être considérées comme de la recherche sous contrat et comme directement dépendantes des relations politiques respectives entre la RDA et la Chine (Krauth/Wolz 1998). Alors que les études sur le Moyen-Orient et sur l'Asie du sud se trouvaient sous contraintes partielles à cause du statu quo respectif des affaires étrangères, elles produisirent aussi un nombre important de recherches valables à l'échelle internationale avant 1980, comme le firent les sciences africaines des plus importants centres de recherche et d'enseignement de Leipzig et Berlin (Rothermund; van der Heyden, in: Krauth/Wolz 1998). Avec la réunification de l'Allemagne, beaucoup de cette tradition de recherche fut perdue soit par la fermeture des centres respectifs ou de parties de départements de recherche (comme pour la philosophie ou la sociologie africaines), soit par une incorporation de celle-ci dans les critères de recherche et d'enseignement tout comme dans la structure universitaire de l'Allemagne occidentalisée, qui n'incluait auparavant pas de départements de science régionale. La recherche entreprise sous forme d'études régionales après 1990 allait cependant suivre le modèle américain expliqué plus haut.

En Allemagne, les études régionales n'ont jamais été aussi généreusement financées ni aussi parfaitement organisées qu'aux États-Unis. La raison de leur institutionnalisation apparut d'abord lors de la prétendue "recherche de transformation", centrée sur l'Europe post-communiste dans son ensemble mais massivement menée par rapport à l'Allemagne de l'est; deuxièmement, et de façon plus importante encore, elle apparut au sein de la stratégie du gouvernement fédéral d'internationaliser la science et la recherche allemandes dans le but de mieux rester actif dans la compétition internationale. Cette stratégie avait aussi nourri l'initiative d'excellence et était considérée comme associée à la mondialisation. Si la première orientation produisit de la recherche en transition et démocratisation de type néo-modernisante (voir Spohn 2006), la seconde et la plus récente rejoignit l'initiative d'excellence en dessinant des structures de recherche destinées à pousser plus loin la reproduction des principales hypothèses de la théorie de la modernisation. Ainsi, dans l'appel pour des propositions de recherche lancé par le ministère fédéral de l'éducation et de la recherche (BMBF) en 2008 , la logique pour "la promotion et le développement futurs des études régionales" se retrouve dans le fait de développer des ressources stratégiques pour la gestion de phénomènes de mondialisation comme les flots migratoires grandissants :

La nécessité d'une connaissance approfondie d'autres régions du monde grandit constamment. À la lumière des processus de mondialisation et de migration, la connaissance des réalités et relations locales et régionales tout comme des réalités 
et relations trans-nationales et trans-culturelles devient de plus en plus importante." (BMBF 2008, ma traduction)

21 Plus précisément, l'initiative s'intéresse à (1) mettre en place un renforcement structurel des études régionales; (2) à développer du contenu aussi bien que des approches méthodologiques et théoriques vis-à-vis des études régionales; (3) à développer de nouvelles formes de coopération entre les études régionales et les disciplines "systématiques" comme l'économie, les sciences sociales, le droit, l'histoire, les sciences naturelles et l'ingénierie dans le but de contrebalancer le rôle joué jusqu'ici par l'histoire et la philologie dans la production de la connaissance sur les régions du monde; et (4) à agrandir la visibilité de ces "loci d'expertise" en Allemagne, lesquels font usage de concepts novateurs (BMBF 2008). Cependant, en se basant sur l'historique de ce qui est maintenant devenue une politique étatique de l'excellence universitaire, l'on constate que les objectifs académiques qui mettent en relief l'expertise régionale et dévalorisent la connaissance historico-philologique au profit des "disciplines systématiques" (c'est-à-dire la science dure), révèlent des buts géopolitiques plus vastes. Dans la course pour les premières places dans la hiérarchie mondiale de la production de la connaissance, reproduire une perspective "noyau" sur la valeur de la connaissance (expertise neutre dans le noyau vs. preuves empiriques dans la périphérie), les méthodes utilisées dans le but de générer la connaissance (science vs. les humanités), tout comme la division intellectuelle du travail parmi les sujets et objets de la production de la connaissance sur une échelle globale (l'occident vs. le reste), deviennent tous des moyens de rattraper le chef de file :

Pour maintenir et améliorer la compétitivité dans le processus de mondialisation, il est essentiel que l'ancienne expertise de l'Allemagne par rapport aux différentes régions du monde soit développée plus après. Il sera impossible de communiquer de façon satisfaisante avec les autres régions du monde tant qu'un choix varié d'expertise n'aura pas été rendu disponible. [...] Alors que les études régionales en Allemagne proposent une grande variété de connaissance sur différentes régions, cultures et pays du monde, quelques disciplines individuelles ont une tendance historique marquée et philologique et leur coopération avec les disciplines systématiques est plutôt sélective." (BMBF 2008, ma traduction)

D'une façon similaire à la ligne de financement de l'initiative d'excellence pour les universités d'élite, les bourses de recherche attribuées sur cette base garantissent un financement sur une période de quatre ans seulement et différent grandement en taille selon l'envergure des propositions soumises. De plus, en dépit de l'existence de centres de "sciences régionales" dans les universités de l'ancienne Allemagne de l'Est, seules sept propositions furent sélectionnées après le premier tour et seules quatre d'entre elles reçurent un financement. L'étendue du soutien de l'état dans ce cas est donc difficilement comparable à l'aide durable et à grande échelle accordée aux études régionales américaines par des supports tels que le Programme international de bourses d'études de la Fondation Ford, qui alloua 270 millions de dollars à 34 universités pour des études régionales et de langues entre 1952 et 1966 ; ou tels que la loi sur l'éducation de la Défense Nationale américaine, promulguée en 1958 en réaction au lancement de la première fusée Spoutnik par l'Union Soviétique un an auparavant, et qui accorda par la suite des financements à 125 départements d'études régionales d'universités américaines, en plus de bourses d'études régionales et de langue pour les étudiants de deuxième cycle (Wallerstein 1997 ; Szanton 2004).

23 En même temps, l'étendue et l'ampleur limitées du contrôle gouvernemental en Allemagne présente les programmes d'études régionales comme étant financés avec 
l'opportunité de mettre en place leur propre programme intellectuel et de choisir de ne pas renforcer les principales suppositions de la théorie de la modernisation dans leur étude des "autres" régions du monde, ni de reproduire les asymétries de la production de la connaissance, lesquelles sont caractéristiques des approches euro-centrées et état-centrées des problèmes examinés. En effet, l'étude des interdépendances mondiales d'inégalités sociales au-delà de l'état-nation, entreprise dans un échange mutuel avec des chercheurs d'Amérique latine plutôt que comme une expertise allemande à propos de l'Amérique latine, est l'objectif explicite du réseau de chercheurs établi à l'institut d'études latino-américaines de l'université libre de Berlin au sein du programme d'études régionales de la BMBF. Dans ce cas en particulier, le sousfinancement chronique de l'éducation supérieure allemande se traduit en liberté académique (au moins partielle). Cependant, remettre en question la logique capitaliste de la production de la connaissance reste un problème de responsabilité individuelle et est contingent tout autant que subordonné à l'excellence du financement, et non de la bourse. Alors que les contestataires s'attachent à définir la rhétorique de l'excellence universitaire comme une construction politique au sein d'un champ de pouvoir asymétrique, académiquement parlant (voir Münch 2007), et les grands réseaux de recherche qu'elle finance comme structurellement inertes et inefficaces, ils ne remettent pas en question la logique plus globale qui encourage les "sociétés de la connaissance" auto-désignées à se lancer dans une course pour les meilleures places dans la hiérarchie mondiale de la production de la connaissance.

\section{Le non-occident(al) et ses avatars : perspectives futures}

Le processus de ré-occidentalisation du système universitaire allemand tel qu'examiné dans cet essai a tendu vers la monopolisation de ressources dans des institutions académiques "d'élite". Une des principales conséquences de ce processus, en plus de la reproduction et du renforcement des inégalités existantes du système éducatif, est celle d'empêcher l'incorporation systématique, bien moins que l'institutionnalisation, d'opinions subaltern(isées).

Comme montré plus haut, l'université occidentale de la fin du xix siècle était européenne occidentale, et comme telle, elle était contemporaine et complice des processus de construction de la nation en Allemagne, France et Angleterre. Dans tous ces cas, la construction minutieuse de langues et littératures nationales ainsi que de récits historiques nationaux, en plus de leur incorporation formelle au parcours académique, faisait partie intégrante des projets politiques d'unification nationale, incluant la production de corps de fonctionnaires pour la maintenance et l'expansion de l'empire colonial de la nation (Mielants 2004: 49). Cela impliquait l'institutionnalisation académique de l'opinion d'élites nationales blanches, mâles et hétérosexuelles au détriment des femmes, sujets coloniaux et des autres ethni(cisés)/ racial(isés) au-delà de leurs propres limites coloniales. Alors que la Grande-Bretagne a vécu au moins une inclusion partielle de la politique de minorité au sein du courant principal, en même temps que l'émergence d'une "industrie de relations entre races" universitaire (Small/Solomos 2006: 250) depuis les années 60, les deux étant dues en partie à l'immigration postcoloniale massive venant du nouveau Commonwealth, la France et l'Allemagne se sont montrées particulièrement inflexibles à cet égard. Le tout 
premier programme d'études de genre en Allemagne a été mis en place en 1997 à l'université Humboldt et a vite été suivi par de nombreux programmes d'études féminines et de genre à travers le pays, mais ceux-ci disposent d'un très petit nombre de postes universitaires ${ }^{7}$ (pour la plupart débutants ou non titulaires). Quant aux études ethniques, jusqu'ici elles sont, au mieux, incorporées aux programmes de recherche sur les migrations ou aux départements de littérature; il n'existe pas de programme d'études queer (le seul en fonctionnement à Hambourg, créé en 2003, a été supprimé cinq ans plus tard); alors même que les études critiques de migration et des recherches nouvelles sur les sexes et l'intersectionalité sont regroupées dans une poignée de chaires en sociologie ou en science politique de la "diversité", ou sont entreprises par des chercheurs free-lance.

Tout comme en France, où le terme "immigration" est utilisé depuis longtemps par procuration pour désigner la race et la maltraitance raciale des immigrés dans les discours universitaires et publics (Balibar 1999), les discussions universitaires et politiques allemandes sur le racisme, les relations raciales et la migration postcoloniale emploient de façon consistante le terme "race" (Rasse) soit entre guillemets soit en tant que néologisme anglais dans des textes écrits en allemand, afin de marquer la différence entre les phénomènes raciaux récurrents et l'utilisation du concept biologique de race par l'Allemagne nazie. Jusqu'à très récemment, la pertinence des discussions autour du racisme, du conflit ethnique et des contextes postcoloniaux tendait généralement à être complètement réprouvée du discours scientifique social grand public à cause du court passé colonial de l'Allemagne, qui ne garantissait apparemment pas d'attention à un présent postcolonial (Gutiérrez-Rodríguez 1999, 2010 ; Boatcă/Costa 2010). Pour les intellectuels critiques allemands, ne disposer (virtuellement) d'aucun programme universitaire touchant aux différences sociales de la perspective des revendications émancipatoires des subalternes, relève non seulement du Sonderweg (chemin particulier) allemand regrettable en comparaison avec d'autres paramètres d'Europe occidentale comme dans les contextes britanniques et scandinaves, mais aussi, et surtout, du décalage temporel et financier par rapport à l'université américaine (Dietze et al. 2007).

Si cette interprétation est correcte, elle pourrait paradoxalement constituer un terreau d'optimisme en regard de développements futurs en Allemagne, et être l'unique circonstance dans laquelle une politique de rattrapage pourrait se montrer bénéfique. Aux États-Unis, ce qui a commencé comme la politique leader, contrôlée par l'état, du renforcement des études régionales dans les années 40 et 50 et qui a subi de sévères attaques dans la période post-1968, a peu a peu laissé place à l'institutionnalisation primaire des départements d'études féminines et ethniques. Nées de la révolte de groupes marginaux contre les structures universitaires dominantes, mais aussi de la radicalisation politique et intellectuelle générée par le contact universitaire avec les ères de recherche (souvent postcoloniales), ces nouvelles entreprises universitaires proposaient des variantes critiques aux études régionales qui se concentraient sur le "Tiers-Monde de l'intérieur" (les minorités ethniques et raciales du Nord) et ses liens avec le sud mondialisé, comme la diaspora noire et les femmes du tiers-monde (Wallerstein 1997). En tant que tels, ils étaient essentiels dans l'ouverture d'un espace institutionnel pour la rencontre entre les Autres ethnicisés et racialisés, en promouvant les perspectives subalternes dans des domaines qui avaient jusque-là été 
monopolisés par des professeurs blancs et des étudiants avalisant les épistémologies européennes (Grosfoguel 2007).

Même si le processus identifié ici comme la re-occidentalisation du système universitaire allemand n'a rien de la success story qu'elle prétend être, elle est parvenue à se rapprocher du modèle américain qu'elle suivait sans le rattraper de façon systématique. Cependant, la politique de rattrapage derrière l'initiative d'excellence a emprunté leurs caractéristiques aux défis lancés envers le système américain dès les années 60 , comme cela apparait clairement dans les discussions sur les programmes d'études régionales et les débuts timides de l'institutionnalisation académique des politiques sur les minorités. Il est vrai que dans le cas américain, l'incorporation de cette dernière dans le champ mondial a entraîné la dépolitisation relative des études féminines et de beaucoup de courants d'études ethniques dans les décennies qui ont suivi. Mais puisque s'embarquer sur le chemin d'un modèle à suivre a jusqu'ici conduit à un Sonderweg avec un résultat différent dans le cas de l'Allemagne (comme dans toute politique modernisationiste), passer des études régionales parfaitement eurocentriques à une structure académique systématique d'études subalternes serait un Sonderweg très intéressant à suivre.

\section{BIBLIOGRAPHIE}

Bibliographie

Balibar, Etienne (1991) : "Racism and Crisis," in : Étienne Balibar/Immanuel Wallerstein, Race, Nation, Class : Ambiguous Identities, New York : Verso, p. 217-227.

BMBF (2009): “Bekanntmachung des Bundesministeriums für Bildung und Forschung von Förderrichtlinien zur Stärkung und Weiterentwicklung der Regionalstudien (area studies), at www.bmbf/foerderungen/13101.php (last accessed April 6, 2010).

Boatcă, Manuela/Costa, Sérgio, “Postcolonial Sociology. A Research Agenda”, In GutiérrezRodríguez, Encarnación/Boatcă, Manuela/Costa, Sérgio (eds.), Decolonizing European Sociology. Transdisciplinary Approaches, Farnham: Ashgate, 2010, p. 13-32.

Darnstädt, Thomas, "Siemens statt Humboldt. Wie Inkompetenz, Finanznot und Verantwortungslosigkeit das Universitätsstudium ruinieren,” Der Spiegel 28, 12.07.2010, p. 40-45.

DFG, "Excellence Initiative at a Glance," available at: http://www.dfg.de/download/pdf/ dfg_im_profil/geschaeftsstelle/publikationen/exin_broschuere_0809_en.pdf (last accessed August 30, 2010), 2008.

DFG, “Excellence Initiative," available at: www.excellence-initiative.com/excellence-initiative (last accessed August 13, 2010), 2010.

Dietze, Gabriele/Hornscheidt, Antje/Palm, Kerstin/Walgenbach, Katharina, Einleitung, In Walgenbach et al. (eds.): Gender als interdependente Kategorie. Neue Perspektiven auf Intersektionalität, Diversität und Heterogenität, 2007, p. 7-22. 
DiMeglio, Mauro, “The Social Sciences and Alternative Disciplinary Models", In Richard E. Lee/ Immanuel Wallerstein (coord.), Overcoming the Two Cultures: Science versus the Humanities in the Modern World-System, Boulder: Paradigm Publishers, 2004, p. 55-72.

Donoghue, Frank, "Wo viel Licht ist, ist auch viel Schatten. Hochschulen in den USA zwischen Elite und Titelmühlen", In Forschung und Lehre 9 (17), 2010, p. 632-635.

Eurostudent, Social and Economic Conditions of Student Life in Europe, Bielefeld: Bertelsmann, 2008.

Goedegebuure, L.C.G., Higher Education Policy: An International Comparative Perspective, Enschede, Netherlands, Centrum voor Studies van het Hoger Onderwijsbeleid, 1993.

Gottschall, Karin/Hagemann, Karen, "Die Halbtagsschule in Deutschland: Ein Sonderfall in Europa?”, In Aus Politik und Zeitgeschichte B41, 2002, p. 12-22.

Grosfoguel, Ramón, “Los dilemas de los estudios étnicos estadounidenses : multiculturalismo identitario, colonización disciplinaria y epistemologias decoloniales", In Universitas Humanística 63, January-June 2007, 2007, p. 35-47.

Gutiérrez-Rodríguez, Encarnación, “Decolonizing Postcolonial Rhetoric”, In Gutiérrez-Rodríguez, Encarnación/Boatcă, Manuela/Costa, Sérgio (eds.), Decolonizing European Sociology.

Transdisciplinary Approaches, Farnham: Ashgate, 2010, p. 49-67.

Helbrecht, Ilse, "The Bologna Process: How the European university is endangered through the creation of a European space of higher education" in Environment \& Planning 39, 2007,

p. 2799-2806.

Hornbostel, Stefan, “Evaluation der Exzellenzinitiative: Gibt es objektive Kriterien für Exellenz?", In Bloch, Roland (ed.), Making Excellence. Grundlagen, Praxis und Konsequenzen der Exzellenzinitiative, Bielefeld: Bertelsmann, 2008, p. 49-63.

Jung, Sabine, “Die ‘Brain Circulation' fördern. Wissenschaftler auf dem Weg zurück nach Deutschland," Forschung \& Lehre 7 (17), 2010, p. 502.

Krauth, Wolf-Hagen/Wolz, Ralf, Wissenschaft und Wiedervereinigung, Asien- und Afrikawissenschaften im Umbruch, Berlin: Akademie, 1998.

Kreckel, Reinhard, "Education, knowledge and social differentiation: new elites and new inequalities?" In Hansgünther Meyer (ed.), Der Dezennien-Dissens. Die deutsche Hochschul-ReformKontroverse als Verlaufsform, Berlin: trafo Verlag, 2006, p. 261-275.

Kreckel, Reinhard, Zwischen universeller Inklusion und neuer Exklusivität. Institutionelle Differenzierungen und Karrieremuster im akademischen Feld: Deutschland im internationalen Vergleich, conference paper, http://www.soziologie.uni-halle.de/kreckel/docs/juniorstaff-t-fertig.pdf , 2008.

Lewis, Martin W./Wigen, Kären E., The Myth of Continents. A Critique of Metageography, Berkeley: University of California Press, 1997.

Manicas, Peter T., “The Social Science Disciplines: The American Model” In Peter Wagner/Björn Wittrock/Richard Whitley (eds.), Discourses on Society: the Shaping of the Social Science Disciplines, Dordrecht: Kluwer Academic, 1991, p. 45-71.

Meyer, Hans Joachim, "Worum geht es im Exzellenzwettbewerb? Eine Kritik", Forschung \& Lehre 8 (17), 2010, pp. 566-568.

Meyer, Hansgünther, "Der Dezennien-Dissens. Begriff und Themen" In Der Dezennien-Dissens. Die deutsche Hochschul-Reform-Kontroverse als Verlaufs-form, Berlin: trafo Verlag, 2006, p. 21-221. 
Mielants, Eric, "Reaction and Resistance: The Natural Sciences and the Humanities, 1789-1945" In Lee, Richard E./Wallerstein, Immanuel (coord.), Overcoming the two cultures. Science versus the Humanities in the Modern World-System, Boulder: Paradigm Publishers, 2004, p. 34-54.

Mignolo, Walter, "Globalization and the Geopolitics of Knowledge. The Role of the Humanities in the Corporate University", Nepantla. Views from South 4.1, 2003, p. 97-119.

Müller, Hans-Peter/Reckwitz, Andreas/Weiß, Anja, Editorial, Berliner Journal für Soziologie 1 (21), 2011, p. 1-5.

Münch, Richard, Die akademische Elite, Frankfurt a.M.: Suhrkamp, 2007.

Paradeise, Catherine/Reale, Emanuela/Goastellec, Gaëlle, In Paradeise, Catherine/Reale, Emanuela/Bleiklie, Ivar/Ferlie, Ewar (eds.), University Governance. Western European Comparative Perspectives, Springer, 2009, pp. 197-225.

Picht, Georg, Die deutsche Bildungskatastrophe, Walter-Verlag, Olten, 1964.

Roche, Mark W., "Motivation und regelmäßige Kontrolle. Die amerikanischen Universitäten zwischen Anreizstrukturen und Rechenschaftspflichten”, Forschung \& Lehre 7 (17), 2010, p. 499-501.

Schimank, Uwe/Lange, Stefan, "Germany: A Latecomer to New Public Management", In Paradeise, Catherine/Reale, Emanuela/Bleiklie, Ivar/Ferlie, Ewar (eds.), University Governance. Western European Comparative Perspectives, Springer, 2009, pp. 51-75.

Small, Stephen/Solomon, John, "Race, Immigration and Politics in Britain: Changing Policy Agendas and Conceptual Paradigms 1940s-2000s", International Journal of Comparative Sociology 47 (3-4), 2006, p. 235-257.

Smolka, Dieter, "Die PISA-Studie. Konsequenzen und Empfehlungen für Bildungspolitik und Schulpraxis", Aus Politik und Zeitgeschichte B41, 2002, p. 3-11.

Spohn, Willfried, "Multiple, Entangled, Fragmented and Other Modernities. Reflections on Contemporary Sociological Research on Europe, North and Latin America", In Costa, Sérgio/ Domingues, José Maurício/Knöbl, Wolfgang/Silva, Josué (eds.), The Plurality of Modernity: Decentring Sociology, Mering, Hampp, 2006, p. 11-22.

Stremlin, Boris, "Constructing Authority: The Rise of Science in the Modern World", In Lee, Richard E./Wallerstein, Immanuel (coord.), Overcoming the two cultures. Science versus the Humanities in the Modern World-System, Boulder, Paradigm Publishers, 2004, p. 9-33.

Szanton, David L., "The Origin, Nature and Challenges of Area Studies in the United States", In David L. Szanton (ed.), The Politics of Knowledge: Area Studies and the Disciplines, University of California Press, 2004, p. 10-11.

Wallerstein, Immanuel, “The Unintended Consequences of Cold War Area Studies”, In Chomsky, Noam (ed.), The Cold War and the University. Toward an Intellectual History of the Postwar Years, New York: The New Press, 1997, p. 195-232.

\section{NOTES}

1. Lewis and Wigen (1997: 51) distinguent un vaste champ de versions de l'Occident, de "l'Occident minimal standard" simplement composé de la Grande-Bretagne, de la France, du Bénélux et de la Suisse, en passant par "L'Occident historique" comprenant tout le royaume médiéval Chrétien autour de 1250 et l'ouest de l'alliance atlantique de 
la guerre froide (le Japon inclus), jusqu'à "l'Occident culturel" regroupé, incluant en plus toutes les zones sous influence et/ou contrôle européens, comme l'Amérique latine et les blancs d'Afrique du Sud.

2. "La fortune des Carnegies, Rockefellers, Cornells, Hopkins, Clarks, Vanderbilts, Standfords, etc., fut utilisée pour construire de nouvelles universités. Mais, de façon tout aussi importante, ces institutions publiques qui les avaient copiées et les vieilles universités "traditionnelles", Yale, Harvard et Columbia, se retrouvèrent alors en compétition quant à leurs étudiants et à leur statut, puis furent prises en main par des entrepreneurs universitaires dont les valeurs et les objectifs étaient intimement liés à l'ordre libéral et entrepreneurial émergeant" (Manicas 1991:48).

3. Dans un débat long d'une décennie, les sociologues allemands ont sérieusement remis en question la cohérence des classes et strates sociales en tant que catégories d'analyse des sociétés occidentales postindustrielles. À ce jour, les euphémismes de classes tels que "origine sociale" sont utilisés à la place du langage de classe conventionnel, intimement associé au marxisme en Allemagne et ainsi théoriquement illégitime pour la vaste majorité des sociologues. Cette situation précise a mené Rainer Geißler (2002: 141) à parler du "Sonderweg allemand de l'analyse de la structure sociale". Pour une exception confirmant la règle plus récente, voir l'édition de mai 2011 du Berliner Journal für Soziologie, notamment l'introduction par Müller et al. (2011).

4. Avec un âge moyen de 26 ans, les diplômés allemands sont les plus vieux diplômés des pays de l'OCDE. Voir Eurostudent 2008, Darnstädt 2010.

5. Pour une analyse fouillée de l'impact de la pression compétitive grandissante sur les systèmes de l'éducation supérieure en Allemagne depuis les années 80, voir Schimank/ Lange 2009.

6. Au sujet des universités américaines d'élite, Donoghue (2010) dit qu'elles sont des "biens de consommation extrêmement coûteux". Il répertorie un chiffre de 35000 \$ pour les frais universitaires en moyenne, comparés à un salaire annuel moyen de 29000 \$ aux États-Unis.

7. De façon criante, seuls $18 \%$ des professeurs d'université en Allemagne sont des femmes. Ce chiffre a récemment été dressé en étendard dans la presse allemande comme un succès remarquable, étant donné qu'il démontre la multiplication par $2 \mathrm{du}$ chiffre de 1999 concernant les professeurs femmes (autour de 9\%). Il a été moins souligné que cette augmentation est en partie due à la nomination de femmes à de nombreux postes de non-titulaires (Juniorprofessur) mis en place depuis 2002. Le fait que le nombre de professeurs d'université recrutés parmi les Autres ethnicisés/ racialisés en Allemagne est de moins de $1 \%$ n'est même pas mentionné dans ce cas.

\section{RÉSUMÉS}

Résumé

Depuis le début des récentes réformes universitaires en Allemagne, la nouvelle division du travail académique a suivie de manière fidèle le model Etatsunien de séparation entre enseignement et recherche en établissant d'une part une «université d'élite » qui jouit de subventions étatiques 
considérables et en réduisant le reste des universités au seul enseignement standard et aux niveaux de Licences -ou au mieux des Masters - où la charge d'enseignement ne permet aucun travail de recherche. La dénommée «Initiative Excellence », processus d'évaluation qui a eut lieu entre 2006 et 2007 et durant lequel 9 Universités - dont l'Université Libre de Berlin, l'université de Munich et celle d'Heidelberg - furent désignées «Universités d'élite », représente jusqu'à présent l'exemple le plus clair et le plus institutionnalisé de politique étatique visant à confiner le travail de recherche dans des institutions spécialisées, dans le sillage des instituts Max Plancks, Leibniz ou des Sociétés Fraunhofer créées dans les années 70 et 80 . Toutefois, alors que ces premières expériences créaient une gamme élargie d'opportunités d'emplois pour les chercheurs, le but de cette communication est de montrer que l' " initiative excellence " représente ce que l'on peut appeler un processus de «commercialisation indirecte " (Reinard Kreckel), un exemple du «capitalisme académique » (Slaughter /Leslie) au sein des Universités d'Europe de l'Ouest qui se fondaient traditionnellement sur les subventions de l'Etat et le contrôle de ce dernier sur les finances de l'institution. A cette fin, nous procéderons en deux étapes : premièrement, en analysant l'idée de Reinhard Kreckel selon laquelle la globalisation néolibérale exerce des pressions économiques et fiscales nouvelles sur les Etats qui se répercutent alors sur les systèmes Universitaires dont ils ont la gestion. Ainsi, nous analyserons comment le discours élitiste de l'éducation supérieure allemande produit de nouvelles formes d'exclusion en lieu et place du système égalitaire et méritocratique vanté à travers la formule des " sociétés de connaissance ». Dans un second temps, au travers d'exemples récents d'appels à candidature pour des bourses du ministère de l'éducation et des centres de recherches financés par l'Etat, nous focaliserons notre étude sur la reprise des Spécialisations disciplinaires comme l'une des cibles majeure de "l'initiative excellence» et de son financement, dans le but de montrer comment le soutien financier de l'Etat envers les recherches sur les inégalités et la démocratisation (dans le contexte des études latino-américaines) et des recherches sur les transformations (dans le contexte des études sur l'Europe de l'est) renforce les principales prévisions des théories de la modernisation et reproduit les asymétries dans la production de connaissances, qui sont caractéristiques des approches eurocentriques et Etatocentriques des solutions en question.

Abstract

In the wake of the recent university reforms, the new academic division of labor in Germany has subserviently followed the U.S.-model of creating a rift between research and teaching universities by establishing on the one hand so-called "elite universities" that benefit from considerable state funding and on the other hand trying to reduce the rest to pedagogical carbon copies of standard curricula that can only award bachelor's or at most master's degrees and where the teaching load allows (almost) no research.

The so-called "excellence initiative", an evaluation process taking place between 2006 and 2007, in the course of which 9 universities - among which the Free University of Berlin, the University of Munich, and the University of Heidelberg - were designated as "elite universities", represents Germany's hitherto clearest and most highly institutionalized example of a state policy aimed at confining research to specialized institutions, such as was already the case with the Max Planck institutes, the Leibniz institutes and the Fraunhofer societies founded in the 1970s and 1980s. However, while earlier steps in this direction only created a wider array of employment options for researchers, the argument of this paper is that the excellence initiative represents what has been called a process of "indirect commercialization" (Reinhard Kreckel) or an instance of "academic capitalism" (Slaughter/Leslie) of/in the Western European university system, which traditionally relied on state financing and state control of university finances. To this end, the paper proceeds in two steps: First, following Reinhard Kreckel's argument that neoliberal globalization exerts new economic and fiscal pressures on the state that are then passed on to 
the higher education systems depending on them, the paper looks at how the new elite discourse of German higher education is producing new structures of exclusion instead of the professed more egalitarian and meritocratic system of self-designated "knowledge societies".

Second, using the examples of recent calls for grant applications by Germany's ministry of education and state-funded research agencies, the paper zooms in on the revival of area studies as one of the prominent targets of the excellence initiative and funding in order to show how strong state financial support for inequality and democratization research (in the context of Latin American studies) and transformation research (in the context of Eastern European studies) reinforces the main assumptions of modernization theory and reproduces the asymmetries of knowledge production characteristic of Euro- and state-centered approaches to the issues in question.

\section{Resumen}

Desde el principio de las recientes reformas universitarias en Alemania, la nueva división del trabajo académico siguió fielmente al modelo estadounidense de división entre enseñanza e investigación al crear por una parte una "Universidad de élite" que goza de considerables subvenciones de Estado y al querer reducir el resto a pedagogías de curriculum/curriculos estandares que solo pueden atribuir grados de licenciaturas, o a lo mejor de maestrías, y donde la carga de enseñanza no permite (casi) ningún trabajo de investigación. La denominada "Iniciativa Excelencia", proceso de evaluación que se desarrolló entre 2006 y 2007 y durante el cual 9 universidades - de las cuales, la Universidad Libre de Berlín, la Universidad de Munich y la de Heidelberg - fueron designadas "Universidades de élite", representa hasta ahora el ejemplo más obvio y el más institucionalizado de política de Estado que confia el trabajo de investigación a instituciones especializadas, como los institutos Max Planck, Leibniz o las sociedades Fraunhofer creados en los años 70 y 80 . Sin embargo, si estas primeras experiencias creaban una gama amplia de oportunidades de empleo para los investigadores, la meta de esta comunicación/presentación es mostrar que la "Iniciativa Excelencia" representa lo que se puede llamar un proceso de "comercialización indirecta" (Reinard Kreckel), un ejemplo de "capitalismo académico" (Slaughter/ Leslie) en universidades de Europa occidental que tradicionalmente contaban con las subvenciones de Estado y el control de este último sobre las finanzas de la institución. Por ende, procederemos en 2 etapas: primero, con el análisis de la idea de Reinhard Kreckel según la cual la globalización neoliberal ejerce nuevas presiones económicas y fiscales sobre los Estados que de igual manera se transmiten a los sistemas universitarios que gestionan. Entonces analizaremos cómo el nuevo discurso elitista de la educación superior alemana produce nuevas formas de exclusión en vez de un sistema igualitario y meritocrático alabado bajo la denominación "sociedades del conocimiento". En un segundo tiempo, a través de ejemplos recientes de convocatorias a concurso para becas del ministerio de educación y de centros de investigación financiados por el Estado, focalizaremos nuestro estudio sobre el resurgimiento de las áreas de estudios como uno de los mayores blancos de la "Iniciativa Excelencia" y de su financimiento. Asi se ve como el apoyo financiero del Estado para la investigaciones sobre desigualdades y democratización (en el contexto de los estudios latinoamericanos) y las investigaciones sobre las transformaciones (en el contexto de los estudios de Europa del este) sustenta las previsiones de las teorías de modernización y reproduce las asimetrias en la producción del conocimiento, lo que es característico de los enfoques eurocéntricos y etatocéntricos de las soluciones en cuestión. 


\section{AUTEURS}

\section{MANUELA BOATCĂ}

Assistant Professor of Sociology at the Latin America Institute of the Freie Universität Berlin. Her research interests include world-systems analysis, sociology of development, gender and violence research, and postcolonial studies in historical-comparative perspective, with a regional focus on Eastern Europe and Latin America. She is author of From Neoevolutionism to World-Systems Analysis. The Romanian Theory of 'Forms without Substance' in Light of Modern Debates on Social Change, 2003, and co-editor of Decolonizing European Sociology. Transdisciplinary Approaches (with

Encarnación Gutiérrez-Rodríguez and Sérgio Costa), Aldershot: Ashgate 2010, as well as of Global, multiple and postcolonial modernities(with Willfried Spohn), Munich 2010.Freie Universität Berlin 